\title{
NEW ACTIVE METHODOLOGIES FOR CRITICAL LEARNING IN THE FIELD OF BIOCHEMISTRY OF HUMAN NUTRITION
}

\author{
Catharina Merchante ${ }^{1}$, David Posé1, Fernando Gallardo ${ }^{1}$, Mar Quiñones ${ }^{2}$, \\ Juan Antonio Gálvez ${ }^{3}$, \& Beatriz Martínez-Poveda ${ }^{1}$ \\ ${ }^{1}$ Department of Molecular Biology and Biochemistry, University of Málaga (Málaga, Spain) \\ ${ }^{2}$ Department of Physiology, University of Santiago de Compostela (Santiago de Compostela, Spain) \\ ${ }^{I}$ Department of Education and Academic Training, My Scientific Company (Spain)
}

\begin{abstract}
Background: The teaching of the subject "Biochemical Basis of Human Nutrition" of the Degree in Biochemistry is based on the premise that students apply the knowledge acquired in previous courses concerning biochemistry and metabolism. However, for many topics covered in this subject, not rigorously application of this knowledge has been detected, existing influences derived from non-expert information available in the media. To a large extent, this problem lies in the fact that nutrition is a topic widely covered in the media, although often in a generalized, incomplete and not very rigorous way.

Methodologies: In this project we proposed students to apply a critical view on nutrition-related information available in the media, with special emphasis on the hottest topics, such as transgenic foods and Mediterranean diet. For this purpose, we designed two strategies: (1) a mini-workshop activity in connection with the subject "Food Biotechnology" focused on the use of transgenic foods; (2) involving students in the creation and management of a web page aimed at dealing with topics related to nutrition, worked from two approaches (informative and scientific). For the development of these activities, students were assigned to different working groups and information about the knowledge of the students in the topics was collected in pre- and post-activity tests. In this way, we involved students in real activities of expert search and screening of information, in order to communicate it in different environments.

Results: The project was developed during two academic years, involving students from two successive promotions (30 students in the first year and 39 in the second year). The activities proposed within the project were voluntary, and the percentage of adherence to them was $100 \%$ in both cases, indicating the high degree of acceptance among the students. We created a website (www.lawebnatural.com) in the context of this project. In the activities aimed at researching and writing articles on specific topics within the web page environment, questionnaires were carried out prior to the development of the activities to evaluate the degree of knowledge that the students had about the topics to be worked on in the activities. The implementation of post-activities questionnaires showed an improvement percentage of $85 \%$ in the knowledge of the topics. The elaboration of graphic material on transgenic foods for the mini-workshop activity, was another profitable activity contributing to a better understanding of the topics.

Conclusions and implications: The use of these dynamics concerning the active participation of students in creative tasks based on information search improves the quality of learning. The choice of current topics in nutrition awakens the students' critical spirit, as they confront their pre-established ideas about these topics with the new knowledge acquired.

Findings: This communication is derived from the Educative Innovation Project PIE19-068, funded by University of Malaga. Websites were supported by funds from My Scientific.
\end{abstract}

Keywords: Nutrition learning, web page creation, critical learning, nutrition topics.

\section{Introduction}

During the course of the Degree in Biochemistry, students progressively acquire many concepts about biochemistry and metabolism, reaching a deep knowledge of them after the first courses. The passage through compulsory subjects common to both Mentions, such as Fundamentals of Biochemistry, Experimental Biochemistry I and II, in the first course, and Regulation of Metabolism in the second course (in addition to other subjects specific to each of the Mentions) ensures that in higher courses 
students can apply the conceptual bases necessary for the acquisition of new knowledge in more applied fields. In the particular case of Biochemical Basis of Human Nutrition (BBHN), an elective subject of the last course of the degree, students must integrate this knowledge by directing it to the concept of human nutrition.

Unlike other topics handled during the degree, nutrition is nowadays on everyone's lips. In the media we always find news related to nutrition (specific sections within morning programs, advertisements and news spread through social networks...). We can say that nowadays nutrition is a topic that is widely handled by the general population and not only by experts. This fact has a positive side, as it raises awareness of certain bad habits or informs about good practices related to nutrition. On the other hand, the generalization of nutrition in so many non-specialized contexts can be a great risk: the excess of information can eventually lead to misinformation (Varela-Mosquera, \& Varela-Moreiras, 2017).

During the teaching of the BBHN course, current concepts arise that arouse great interest but that are very much associated with the "vox populi" controversy, and in many cases the students themselves who participate in the course use arguments that are not well contrasted and do not properly integrate the expert knowledge they have acquired during the degree. This has been particularly observed in concepts related to transgenic foods, despite being a topic they have worked on in different subjects. In order to solve this tendency regarding this and other "hot-topics" related to nutrition, we propose 3 objectives focused to encouraging critical thinking in the students and also to explore the use of popular media as a motivational tool for students.

\section{Design}

The present work has been applied to two groups of students (30 and 39 students), enrolled in the subject BBHN during two successive academic years (2019/2020 and 2020/2021). The activities developed were offered on a voluntary basis. The proposed methodology (described in Methods section) was applied during the 4 months of the course, and included the development of different learning methodologies: (1) organizing a mini-workshop format activity in connection with the subject Food Biotechnology, in which the two groups of students assume roles of critical transmitters/receivers of information (focused on the use of transgenic foods); (2) involving students in the creation and management of a web page aimed at dealing with topics related to nutrition, worked from two approaches (informative and scientific). In this way, we intend to involve students in real activities of expert search and screening of information, in order to communicate it in different environments. Questionnaires were prepared about different topics in nutrition, in order to evaluate before and after the training actions, the students' previous knowledge and their general impressions about them. These questionnaires served as a tool to measure the impact of the actions on learning.

\section{Objectives}

1. To encourage a critical view of "hot-topics" related to human nutrition. Biochemistry.

2. Create spaces for communication between subjects of the different Mentions of the Degree in

3. Motivate student participation in outreach media beyond the scope of the subjects.

\section{Methods}

\subsection{Selection of "hot-topics"}

A brainstorming activity was carried out in class, in which students suggested topics related to nutrition that are usually treated in the media or generate doubts in the students themselves as to their veracity or scientific basis. Concepts related to Mediterranean Diet and transgenic foods were included in these topics.

\subsection{Search for information}

Working groups of 3-4 students were established and "hot-topics" were assigned to each group ( 2 topics/group). A dynamic was carried out in class oriented to the search for information in specialized databases (MedLine, Scopus...) or in web pages of official organizations or agencies of international prestige that offer accurate information on topics related to nutrition (FAO...). 


\subsection{Screening and use of the information}

Once the information was compiled, each group screened it, selecting the essential information related to each topic. This activity was carried out by the working groups outside the classroom, and a summary of the essential points and the basic bibliography was elaborated and sent to the teachers for their supervision. The groups of students used the selected information for the elaboration of (1) a poster in congress format. The work was mainly synthetic, since it was essential that all the information collected was structured in an orderly, visual and understandable way to a receiver who does not have much knowledge about the topic. (2) two informative texts referring to each of the two assigned topics. One of the texts was treated from a scientific point of view, explaining in detail the biochemical/metabolic/physiological bases of the topic worked on. The other text was elaborated in a more informative format, trying to convey to the reader the essential ideas about the topic worked on, but without falling into technicality. In total, each group elaborated 4 texts, two scientific and two informative, about the two topics assigned to them. These articles were included in the section of contents in a web page created for the subject.

\section{Results and discussion}

The activities proposed within the project were voluntary, and their adherence to them was an indicator of the students' interest in doing other types of activities to complement lecture attendance. In the two consecutive courses in which they were carried out, the percentage of adherence was $100 \%$, indicating the high degree of acceptance among the students. Moreover, the number of students enrolled in the BBHN course increased by $30 \%$ (from 30 to 39 students) in the second year since the project was launched, from which it can be inferred that the course had an increase in the degree of acceptance among students, since BBHN is an elective subject.

We created a website (www.lawebnatural.com) in the context of this project. The students worked on the different topics, in their more scientific vision and in their divulgative version, within the context of this web page, creating content that in turn motivated their work in the subject and their knowledge of the topics covered. The existence of an open work environment on the Internet, with the possibility of visualization that their work entailed, motivated the students to research the subjects, demonstrating a high degree of adherence to the tasks, with very positive perception.

The elaboration of graphic material on transgenic foods for the mini-workshop activity, and its subsequent sharing by the students, improved their knowledge on this specific topic, which had not been previously worked on in any subject of the Degree in Biochemistry taken by them. Questionnaires were carried out prior to the development of the activities to evaluate the degree of knowledge that the students had about the topics to be worked on in the activities, and were completed with questionnaires carried out afterwards. As a result, an average improvement percentage of $85 \%$ was achieved, demonstrating that the work carried out by the groups of students improved their knowledge of the topics worked on.

\section{Conclusions}

The use of these dynamics that depart from the classic master class model demonstrate that the active participation of students in creative tasks based on information search improves the quality of learning. The choice of current topics in nutrition for the development of the tasks awakens the students' critical spirit, as they confront their pre-established ideas about these topics with the new knowledge acquired.

\section{References}

Varela-Mosquera, G, \& Varela-Moreiras, G. (2017). Introducción a la historia de la nutrición. In A Gil Hernández \& F Sánchez de Medina Contreras (Eds.), Tratado de nutrición, Tomo I (1-18). Editorial Médica Panamericana ISBN:.978-84-9110-190-1 